\title{
On the formation and evolution of magnetic chemically peculiar stars in the solar neighborhood
}

\author{
H. Pöhnl, E. Paunzen, and H. M. Maitzen
}

Institut für Astronomie der Universität Wien, Türkenschanzstr. 17, 1180 Wien, Austria

e-mail: ernst.paunzen@univie.ac.at

Received 20 April 2005 / Accepted 19 June 2005

\section{ABSTRACT}

In order to put strict observational constraints on the evolutionary status of the magnetic chemically peculiar stars (CP2) of the upper main sequence, we have investigated a well established sample of galactic field CP2 objects within a radius of $200 \mathrm{pc}$ from the Sun in the (X, $Y$ ) plane. In total, 182 stars with accurate parallax measurements from the Hipparcos satellite were divided into $\mathrm{Si}, \mathrm{SiCr}$ and $\mathrm{SrCrEu}$ subgroups based on classification resolution data from the literature. Primarily, it was investigated if the CP2 phenomenon occurs at very early stages of the stellar evolution, significantly before these stars reach $30 \%$ of their life-time on the main sequence. This result is especially important for theories dealing with stellar dynamos, angular momentum loss during the pre- as well as main sequence and stellar evolutionary codes for CP2 stars. For the calibration of the chosen sample, the well-developed framework of the Geneva 7-color and Strömgren $u v b y \beta$ photometric system was used. We are able to show that the CP2 phenomenon occurs continuously at the zero age main sequence for masses between 1.5 and $4.5 M_{\odot}$. The magnetic field strengths do not vary significantly during the evolution towards the terminal age main sequence. Only the effective temperature and magnetic field strength seem to determine the kind of peculiarity for those stars. We found several effects during the evolution of CP2 stars at the main sequence, i.e. there are two "critical" temperatures where severe changes take place. There is a transition between $\mathrm{Si}$, $\mathrm{SiCr}$ and $\mathrm{SrCrEu}$ stars at $10000 \mathrm{~K}$ whereas a significant decrease to almost zero of evolved SrCrEu objects with masses below $2.25 M_{\odot}$ at $8000 \mathrm{~K}$ occurs. These conclusions have to be incorporated into models that simulate the stellar formation and evolution of stars between 1.5 and $4.5 M_{\odot}$ in the presence of strong magnetic fields.

Key words. stars: chemically peculiar - stars: evolution - stars: fundamental parameters

\section{Introduction}

In Pöhnl et al. (2003) we have shown that young magnetic chemically peculiar stars (CP2 herafter, according to the notation by Preston 1974) of the upper main sequence exist in young open clusters (ages between 10 and 140 Myr) of our Milky Way. Our result received significant support by the detection (Bagnulo et al. 2003) of a $14.5 \mathrm{kG}$ magnetic field for HD 66318 in NGC 2516 which was also an object of the study by Pöhnl et al. (2003).

The strong stellar magnetic fields of these objects together with the diffusion of chemical elements depending on the balance between gravitational pull and uplift by the radiation field through absorption in spectral lines manifests in strong overabundances for heavy elements such as silicon, chromium, strontium and europium compared to the Sun.

The most important aspect for CP2 phenomenon is the origin of the global stellar magnetic fields for those objects. Two theories have been developed in this respect (Moss 1989):

- The dynamo theory based on the existence of a contemporaneous dynamo operating in the convective core of the magnetic stars. This means that the magnetic fields should only appear at evolutionary stages between the zero and terminal age main sequence (ZAMS and TAMS hereafter), preferably closer to the TAMS.

- The fossil theory has two variants: the magnetic field is either the slowly decaying relic of the frozen-in interstellar magnetic field or of the dynamo acting in the pre-main sequence phase. However, according to this model, strong magnetic fields should be present when a star reaches the ZAMS.

Several divergent interpretations of the evolutionary status and their implication for magnetic field theories of CP2 stars can be found in the literature. We have summarized them in more detail in Pöhnl et al. (2003).

Our main goal for this paper is to investigate the evolutionary status for the CP2 group in the solar neighborhood and to answer the question raised by Hubrig et al. (2000) who stated that 1) the distribution of CP2 stars of masses below $3 M_{\odot}$ in the Hertzsprung-Russell-Diagram (HRD hereafter) differs from that of the "normal" stars in the same temperature range at a high level of significance: magnetic stars are concentrated toward the center of the main sequence band and 2) magnetic 
fields appear only in stars that have already completed at least approximately $30 \%$ of their main sequence life-time.

We have investigated three subgroups ( $\mathrm{Si}, \mathrm{SiCr}$ and $\mathrm{SrCrEu}$ ) of $\mathrm{CP} 2$ stars which are unambiguously classified and have accurate parallax measurements. This sample includes 182 objects within a radius of $200 \mathrm{pc}$ from the Sun in the $(X, Y)$ plane. The astrophysical parameters of these objects were derived taking into account the peculiar characteristics and all results have been carefully compared with already published values.

\section{Program stars}

The target selection for this analysis is based on two main criteria: 1) CP2 stars with known spectral characteristics and 2) Accurate parallax measurements available. We have taken the lists of Renson (1991), Gómez et al. (1998), Renson \& Catalano (2001), Bychkov et al. (2003), Skiff (2003) and Paunzen et al. (2005) to search for well-established CP2 stars. We have only included stars that are unambiguously classified within these references.

From this first list, objects were selected for which the absolute error of the parallax is less than $17.5 \%$ which is the upper limit for applying a correction as suggested by Lutz \& Kelker (1973). Our final list includes 182 objects which were divided into $\mathrm{Si}(61), \mathrm{SiCr}$ (34) and $\mathrm{SrCrEu}$ (87) stars. These are the three subgroups of the CP2 objects that are described in more detail by Preston (1974) and Stepien (1994). The phenomenology is due to classification resolution spectra in which different lines of several elements are more pronounced than in normal objects of the same effective temperature. Even if these subgroups are investigated with high resolution spectra, the characteristic abundance pattern is still different (Wolff 1983).

Figure 1 shows the spatial distribution of our sample. All objects are within a radius of $200 \mathrm{pc}$ from the Sun in the $(X, Y)$ plane.

\section{Determination of the evolutionary status for the CP2 stars}

In the following subsections we describe the method to derive the $\log L / L_{\odot}$ and $\log T_{\text {eff }}$ values for our program stars in more detail. Special care was taken to include and correct the effects of the spectral peculiarities when deriving the age as well as mass of the individual stars.

\subsection{Reddening and metallicity}

Our program stars are located within a radius of $200 \mathrm{pc}$ of the Sun in the $(X, Y)$ plane (Fig. 1) for which the interstellar reddening is not negligible any more. The most common way to derive the reddening for single stars is to apply standard relations within the Strömgren $u v b y \beta$ photometric system (Crawford 1975, 1979; Hilditch et al. 1983). These relations have to be used with caution when applied to CP2 stars because all calibrations are primarily based on the $\beta$ index. Due to variable strong magnetic fields, the $\beta$ index can give erratic

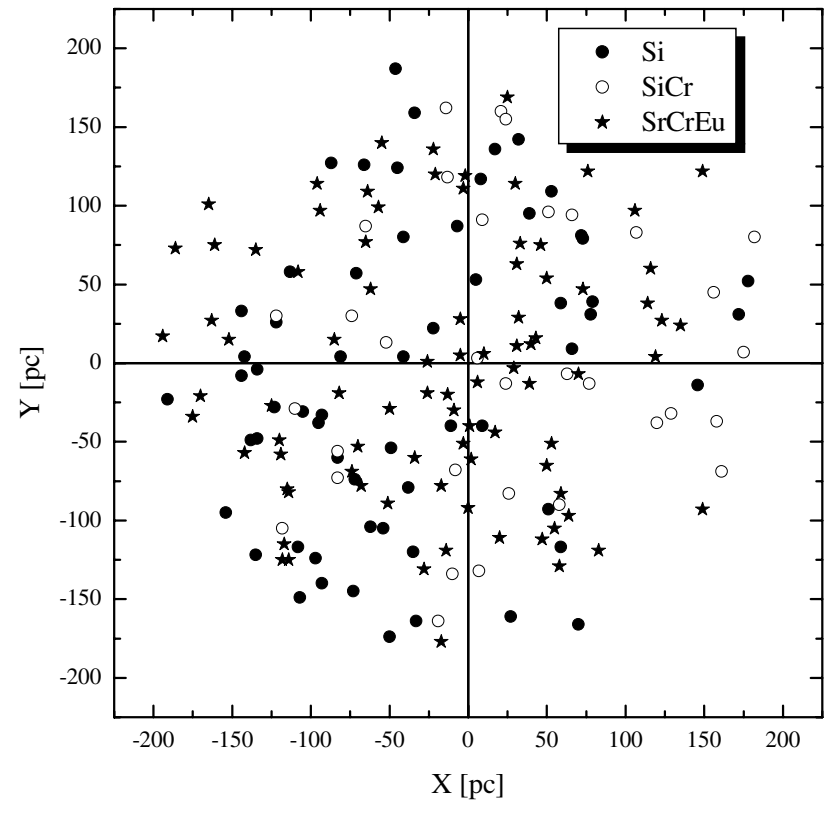

Fig. 1. The spatial distribution of the investigated sample of CP2 stars divided into $\mathrm{Si}$ (filled circle), $\mathrm{SiCr}$ (open circle) and $\mathrm{SrCrEu}$ (asterisk) objects.

values (Catalano \& Leone 1994). Another effect taken into account is a "blueing" effect which manifests in bluer colors due to stronger UV absorption than in normal type stars which can be erratically interpreted as strong reddening (Adelman 1980).

We have therefore carefully checked the reddening values from the Strömgren $u v b y \beta$ photometric system with other sources.

As a first approximation, we have used a "standard" value of $0.25 \mathrm{mag} \mathrm{kpc}^{-1}$ derived from open clusters (Dutra \& Bica 2000). These values were compared with the reddening laws listed by Chen et al. (1998). As the authors note, the reddening for very close objects can be overestimated. We found that for several objects the reddening derived from Chen et al. (1998) has to be multiplied by a factor of 0.7 to be comparable to the other sources.

As a final step, we have compared and averaged the values from all sources. As expected, many stars exhibit no reddening at all.

The metallicity has a severe influence on the determination of the age and mass but only marginally affects the effective temperature (Glagolevskij 1994). Classical CP2 stars are true Population I objects with intrisic solar abundances. Their peculiarities are restricted to the surface only (Nishimura et al. 2004). It is therefore justified to use solar isochrones for the determination of the age and mass (Gómez et al. 1998), but what metallicity should be chosen for the solar neighborhood? Schaller et al. (1992) give $[\mathrm{Z}]=0.0188$ whereas Allende Prieto et al. (2004) found that the solar neighborhood would be "metal-weak" compared to the Sun by 0.06 to 0.11 dex. We have therefore used a value of $[\mathrm{Z}]=0.016$ for the isochrones which were interpolated within the grids by Schaller et al. $(1992,[Z]=0.02)$ and Schaerer et al. $(1993,[Z]=0.008)$. 


\subsection{Effective temperature and luminosity}

For the calibration of the effective temperature, we have made use of the Geneva 7-color photometric system (Golay 1980). All photometric measurements were taken from the General Catalogue of Photometric Data (GCPD, Mermilliod et al. 1997).

The approach by Hauck \& Künzli (1996) was followed. For hot CP2 stars $\left(T_{\text {eff }}>9500 \mathrm{~K}\right)$, the effective temperature is given as

$T_{\text {eff }}=-230+0.941 \cdot T(X, Y)$

where $T(X, Y)$ has to be calibrated within the grids by Künzli et al. (1997). For cooler objects, we have used the calibration by Hauck \& North (1993) which is based on $(B 2-G)_{0}$. For the few cool objects without available Geneva colors, we have used the Strömgren $u v b y \beta$ photometric calibration by Napiwotzki et al. (1993) which is based on the reddening free $\left[c_{1}\right]$ index

$5400 / T_{\text {eff }}=0.2489+0.2698 \cdot\left[c_{1}\right]$.

A comparison of the results for both calibrations of other CP2 stars in the same temperature range shows no systematic trend which is in line with the results by Glagolevskij (2002).

The estimation of the errors for such a calibration has been widely discussed in the literature (Smalley \& Kupka 1997). Hauck \& Künzli (1996), for example, list a statistical error for the photometric calibration of a large sample of normal type stars of $\pm 386 \mathrm{~K}$ depending on the temperature range. North (1998) investigated especially CP2 stars and found an error of the mean of $4.4 \%$ and $3.4 \%\left(\Delta \log T_{\text {eff }}=0.019\right.$ and 0.014$)$ for objects hotter and cooler than $9500 \mathrm{~K}$, respectively. We were not able to compare our calibrated effective temperatures with those used by Gómez et al. (1998) because the latter are not available.

The luminosity calibration for CP2 stars is also affected by stronger UV absorption which results in correction factors $\delta_{B C}$ of up to $0.3 \mathrm{mag}$ for the estimation of bolometric magnitudes for this group (Lanz 1984; North 1998). The luminosity can be calculated as:

$\log L / L_{\odot}=0.4 \cdot\left(4.72-M_{V}-B C+\delta_{B C}\right)$

with

$M_{V}=m_{V}+5 \cdot \log \pi+5-3.1 \cdot E(B-V)$.

The values for the bolometric correction $B C$ and the standard solar value were taken from Flower (1996). Finally, we have corrected for the "Lutz-Kelker effect" (Lutz \& Kelker 1973) which corrects for the bias in the absolute magnitude of a star as estimated from its trigonometric parallax. These corrections are rather small compared to the error of the parallax measurements from Hipparcos at distances up to $200 \mathrm{pc}$ from the Sun.

\subsection{Age and mass}

We have used interpolated isochrones with $[\mathrm{Z}]=0.016$ from Schaller et al. (1992) and Schaerer et al. (1993) for the determination of the ages and masses for the program stars.
The location of a star within the HRD is unambiguously defined by the hydrogen concentration in the core $X_{\mathrm{C}}$ and the stellar mass. The interpolation of these two parameters within the isochrones has been described in Pöhnl et al. (2003).

The knowledge of $X_{\mathrm{C}}$ allows us to determine the relative age $\tau / \tau_{\mathrm{HR}}$, where $\tau_{\mathrm{HR}}$ denotes the time a star is on the main sequence. This is because $\log L / L_{\odot}$ and $\log M / M_{\odot}$ are, at any stage of the stellar evolution, well correlated with $X_{\mathrm{C}}$ Schaller et al. (1992) list value of $X_{\mathrm{C}}=0.68$ and 0.03 for the ZAMS $\left(\tau / \tau_{\mathrm{HR}} \approx 0\right)$ and the TAMS $\left(\tau / \tau_{\mathrm{HR}}=1\right)$, respectively. These values are almost independent of the stellar mass.

Our quadratic interpolation within the isochrones results in:

$\tau / \tau_{\mathrm{HR}}=0.015+2.714 \cdot\left(0.68-X_{\mathrm{C}}\right)-1.845 \cdot\left(0.68-X_{\mathrm{C}}\right)^{2}$.

The statistical error for the complete sample, only taking into account the errors within the interpolation grid, is less than $\pm 5 \%$ for the relative age. The errors for individual objects can be larger due to the errors of the effective temperature (error of the statistical mean is less than $4.4 \%$, see Pöhnl et al. 2003) and the luminosity (determined be the parallax error which is always smaller than $17.5 \%$ ). But throughout this paper, only statistical samples are used instead of values for individual CP2 stars which implies that the statistical error of less than $\pm 5 \%$ for the relative age is justified.

\section{Results}

In Fig. 2 the location of the program stars within the HRD is shown. We have included evolutionary tracks from 1.5 to $4 M_{\odot}$, lines of equal $\tau / \tau_{\mathrm{HR}}$, the ZAMS and TAMS for $[\mathrm{Z}]=0.016$ and the typical error bars. At first sight it is evident that the CP2 stars occupy the whole width of the main sequence which means that they are luminosity class $\mathrm{V}$ objects.

We performed a detailed statistical analysis of the relative ages $\tau / \tau_{\mathrm{HR}}$ for the three samples of $\mathrm{Si}, \mathrm{SiCr}$ and $\mathrm{SrCrEu}$ objects. For this purpose we further divided those samples according to the masses into four subsamples ranging from below $2 M_{\odot}$ to masses higher than $4 M_{\odot}$.

Figure 3 shows the result graphically. The different groups were binned with bin sizes of 0.2 for $\tau / \tau_{\mathrm{HR}}$. A smaller bin size is not justified because of the overall errors and the additional introduction of small number statistics. From Figs. 2 to 4 we are able to conclude:

- The CP2 phenomenon occurs already at the ZAMS. The percentage of all investigated CP2 stars with $\tau / \tau_{\mathrm{HR}}<$ 0.2 is $14.4 \%$ as well as $20.8 \%$ for objects below and above $3 M_{\odot}$ which accounts for $16 \%$ (or 29 stars) of the complete sample.

- The magnetic field strengths do not vary significantly during the main sequence evolution.

- The age distribution of objects below (no clear trend with the age) and above (clear peak at $\left.0.6<\tau / \tau_{\mathrm{HR}}<0.8\right) 3 M_{\odot}$ is statistically different. The Spearman rank order correlation coefficient and the $\chi^{2}$ test (Rees 1987) for these two samples both give a significance level of about $25 \%$ for a possible correlation. This result has to be taken with caution because only five data points are include in this analysis. 


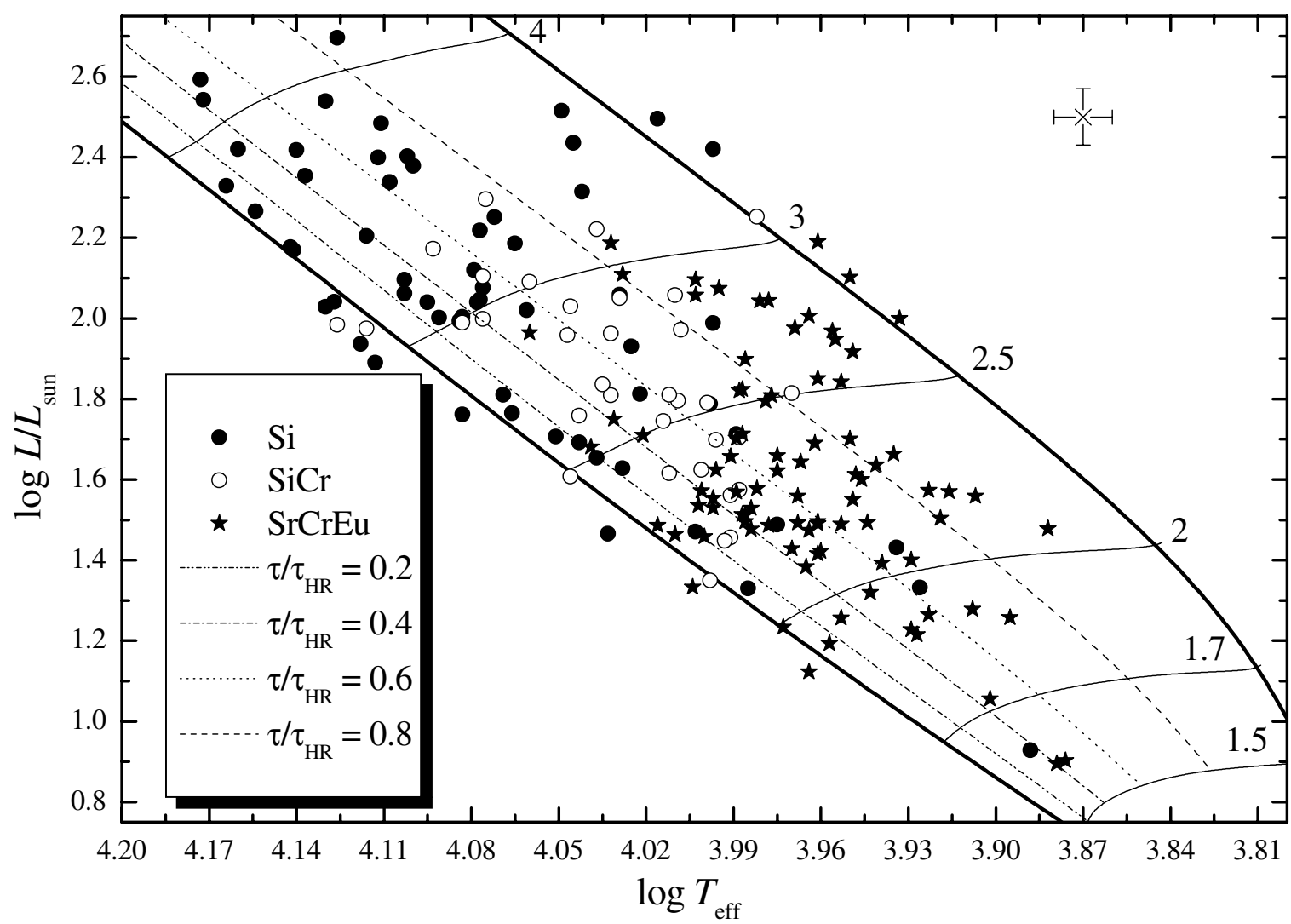

Fig. 2. The Hertzsprung-Russell-Diagram for our sample of CP2 stars, the symbols are the same as in Fig. 1. The evolutionary tracks from 1.5 to $4 M_{\odot}$, lines of equal $\tau / \tau_{\mathrm{HR}}$, the ZAMS as well as TAMS are for $[\mathrm{Z}]=0.016$ taken from Schaller et al. (1992) and Schaerer et al. (1993). The typical errors are plotted in the upper right corner.

- The temperature and the magnetic field strength are the main parameters that determine the manifestation of the chemically peculiarity, i.e silicon - chromium - strontium and europium (from hotter to cooler temperatures).

- Si stars: they can be found within the whole mass range, but more so at young relative ages with higher masses. Their incidence seems to decrease with age except for a peak at between $0.6<\tau / \tau_{\mathrm{HR}}<0.8$. We are not able to decide whether this is due to poor number statistics or a physical effect.

- SiCr stars: most of these objects have masses between 3 and $4 M_{\odot}$. They seem to be transition objects between $\mathrm{Si}$ and $\mathrm{SrCrEu}$ stars. There is, for example, an apparent edge at $\left[\log T_{\text {eff }}, \log B_{\mathrm{e}}\right]$ defined by [4.0, 2.5] and [4.15, 3.5] which clearly separates $\mathrm{Si}$ and $\mathrm{SiCr}$ stars. The age distribution clearly peaks between $0.4<\tau / \tau_{\mathrm{HR}}<0.6$.

- SrCrEu stars: these are the low mass CP2 stars $(M<$ $3 M_{\odot}$ ) with an upper limit of the effective temperature between 9500 and $10000 \mathrm{~K}$. There is a clear negative correlation between the incidence and the age. However, at least $24 \%$ of all objects have relative ages below 0.4 .

The question arises if the age distribution of CP2 stars with masses below $3 M_{\odot}$ is significantly different from that of normal type objects in the same spectral range as suggested by Hubrig et al. (2000). They have used all apparent single type objects of the Bright Star Catalogue as a comparison which resulted in 412 objects. However, their CP sample includes only 33 magnetic stars which is most certainly biased by lower rotational velocities than for the normal type stars (Abt \& Morell 1995). The comparison of such widely different samples has to be treated with caution. We have chosen to follow the approach by Paunzen et al. (2002) who used the sample of the program stars to choose a random sample of normal type objects by matching the effective temperature distribution. They found for stellar masses below $2.7 M_{\odot}$ a peak of the relative age distribution for $0.6<\tau / \tau_{\mathrm{HR}}<0.9$ (see Fig. 2, Paunzen et al. 2002). We found exactly the same behaviour for a random sample of normal type objects within a radius of $200 \mathrm{pc}$ of the Sun in the $(X, Y)$ plane which matches the sample of CP2 stars with masses below 3.0 $M_{\odot}$. We therefore conclude that the different age distribution of low mass CP2 stars as discussed by Hubrig et al. (2000) is due to a bias of the selection criteria and does not reflect an astrophysical process.

North (1993) speculated whether a star can be born as a He-weak and "die" as a Si star, or, similarly, whether a Si star can become a $\mathrm{Cr}$ or a $\mathrm{SrCrEu}$ while evolving. From Fig. 3 it is obvious that there is a surplus of $\mathrm{SrCrEu}$ objects near the TAMS. According to the models by Schaller et al. (1992), an object with $2.5 M_{\odot}$, has at the ZAMS an effective temperature of about $11150 \mathrm{~K}$, after $585 \mathrm{Myr}$ it reaches the TAMS with an effective temperature of $8160 \mathrm{~K}$. During the life time on the ZAMS, the stellar atmosphere changes due to rotational and magnetic effects as well as due to the diffusion mechanism. These effects seem to manifest in the HRD. There is a 


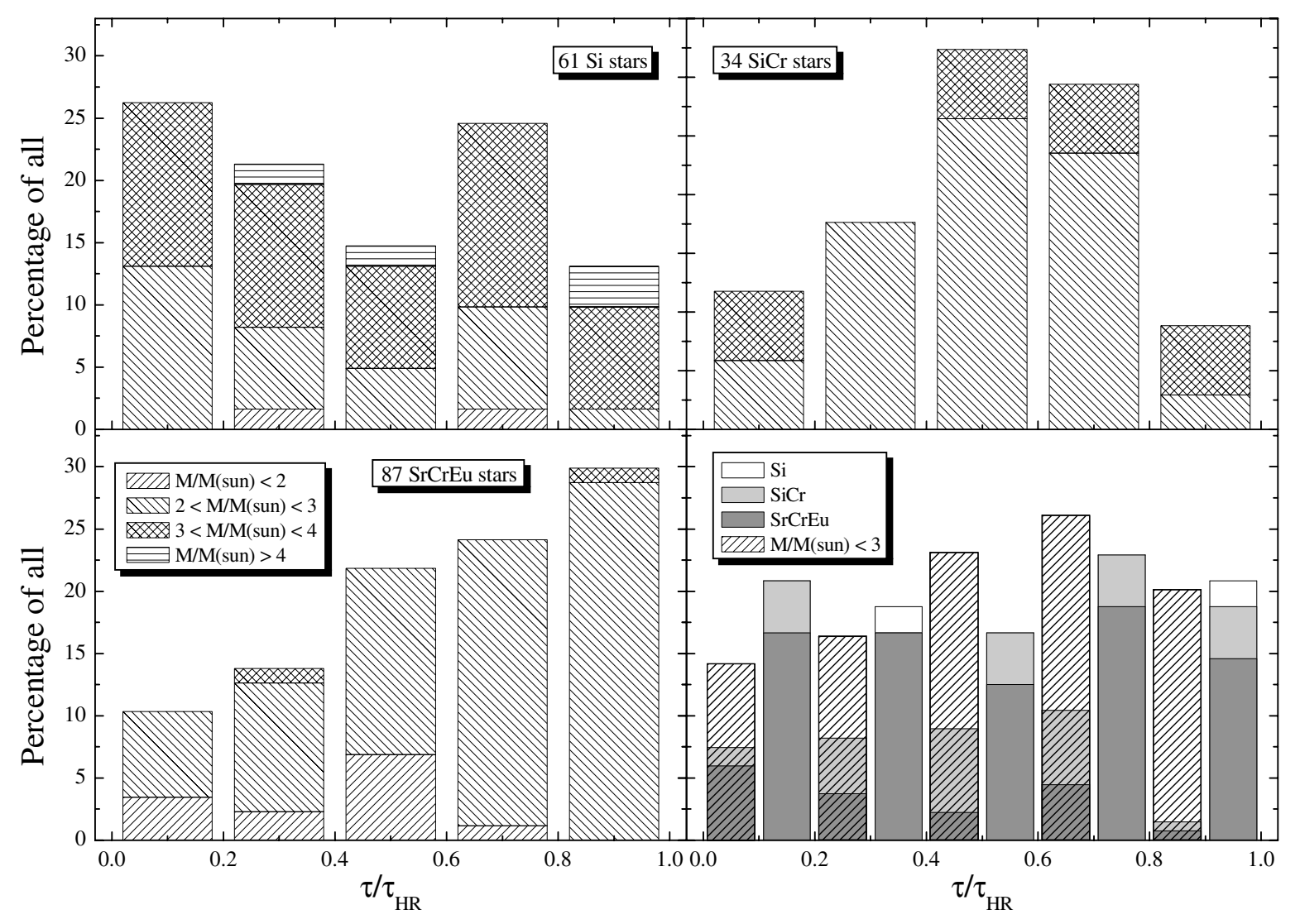

Fig. 3. A detailed statistical analysis of our sample. We have divided the sample into Si (upper left), $\mathrm{SiCr}$ (upper right) and $\mathrm{SrCrEu}($ lower left) subgroups and investigated the incidence of these objects depending on their masses. The lower right panel shows the comparison of all stars divided into subgroups with masses higher (denoted with a pattern) and lower than $3 M_{\odot}$ according to the investigated sample by Hubrig et al. (2000). The Spearman rank order correlation coefficient and the $\chi^{2}$ test for these two samples give both a significance level of about $25 \%$ for a possible correlation. However, these tests are only based on five data points.

dramatic decline of evolved $\mathrm{SrCrEu}$ objects for masses lower than $2.5 M_{\odot}$ whereas it is the opposite case for masses higher than that. There are hardly any evolved Si stars but many evolved $\mathrm{SrCrEu}$ objects. We therefore conclude that there are three different effects:

1. Si stars change their peculiarity as they evolve towards temperatures cooler than $10000 \mathrm{~K}$ on the main sequence.

2. There is an obvious transition between $\mathrm{Si}, \mathrm{SiCr}$ and $\mathrm{SrCrEu}$ objects at $10000 \mathrm{~K}$

3. During the evolution. of CP2 stars with masses lower than $2.25 M_{\odot}$, there is a significant decrease of the incidence for peculiar objects at $8000 \mathrm{~K}$.

\section{Conclusions}

We have investigated a sample of 182 CP2 stars for which homogeneous classifications and accurate parallax measurements are available. The sample was further divided into $\mathrm{Si}, \mathrm{SiCr}$ and $\mathrm{SrCrEu}$ objects, the three main groups. For all program stars effective temperatures, luminosities and thus ages as well as masses were calibrated with the help of standard evolutionary models. For this purpose we used data and calibrations within the Geneva 7-color and Strömgren uvby $\beta$ photometric system. Special care was taken to include the appropriate corrections for the spectral and thus flux peculiarities.
The evolutionary status of $\mathrm{CP} 2$ stars is especially interesting because it still not clear whether these objects show their peculiar nature soon after arriving at the ZAMS or after about $30 \%$ of their life time on the main sequence. Answering this question from the observational side has a direct impact on the theories about the origin of the strong stellar magnetic fields. One model describes the survival of frozen-in fossil fields originating from the medium out of which the stars were formed, the other one follows the idea that a dynamo mechanism is acting in the interior of these stars.

From the location of our program stars we are able to conclude that the CP2 phenomenon occurs already at the ZAMS. At least $16 \%$ of the investigated CP2 stars have relative ages below $20 \%$. We find no significant variation of the magnetic field strengths over the whole main sequence. The effective temperature and the magnetic field strength are the main parameters that determine the kind of the chemical peculiarity, i.e silicon - chromium - strontium and europium (from hotter to cooler temperatures).

The difference in the samples of CP2 stars with masses above and below $3 M_{\odot}$ reported by Hubrig et al. (2000) seems to be a selection and thus bias effect of the peculiar and normal sample.

We find a strong decline of evolved $\mathrm{SrCrEu}$ objects for masses lower than $2.5 M_{\odot}$, whereas there are few evolved 


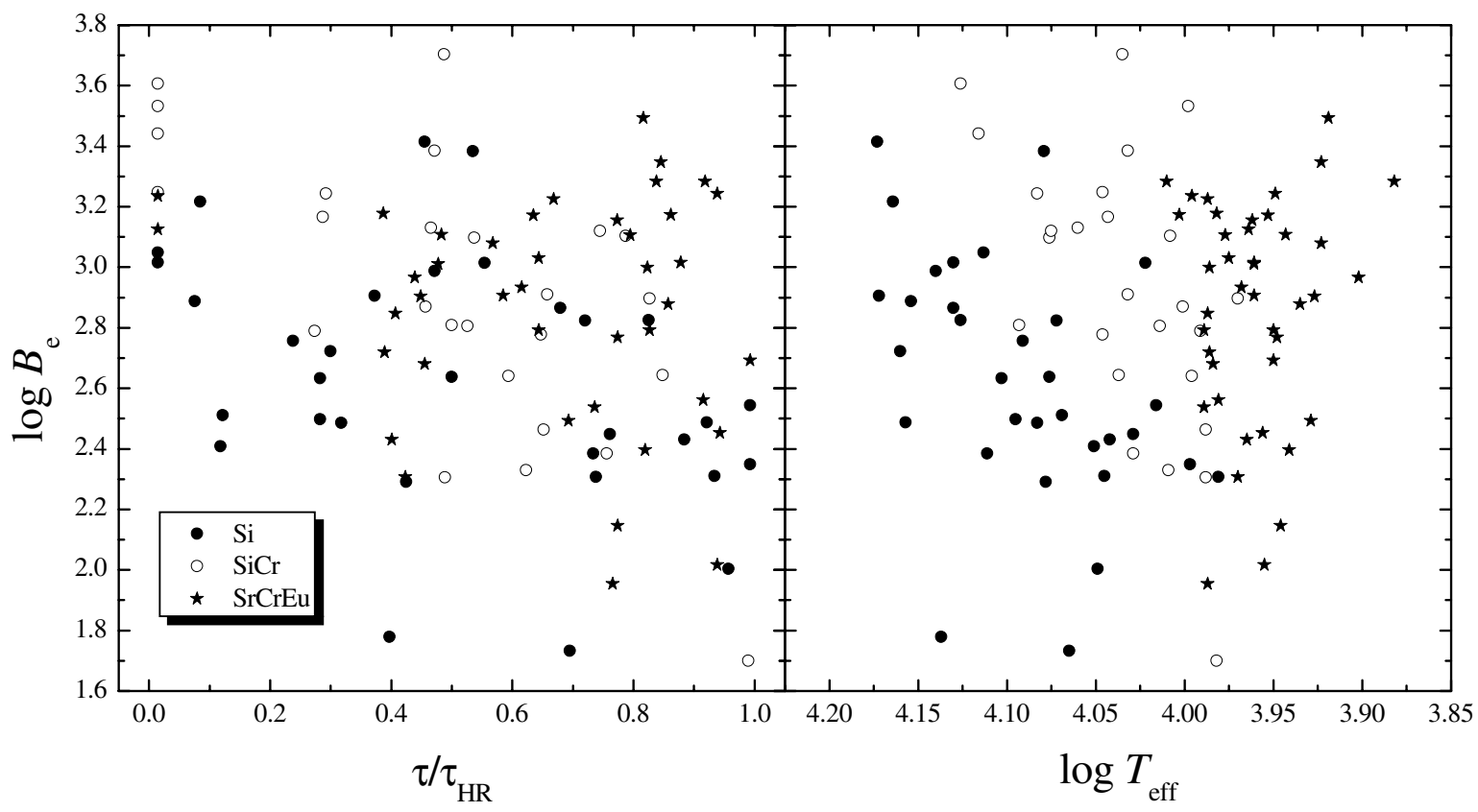

Fig. 4. The $\log B_{e}$ versus $\tau / \tau_{\mathrm{HR}}$ as well as $\log T_{\mathrm{eff}}$ diagrams for our program stars. The magnetic field strengths were taken from Bychkov et al. (2003).

Si stars but many evolved SrCrEu objects above this stellar mass. This can be interpreted as Si stars changing their peculiarity as they evolve at temperatures cooler than $10000 \mathrm{~K}$ which results in a transition between $\mathrm{Si}, \mathrm{SiCr}$ and $\mathrm{SrCrEu}$ objects at that point. Furthermore, the incidence of CP2 stars with masses lower than $2.25 M_{\odot}$ decrease to almost zero below effective temperatures of $8000 \mathrm{~K}$.

These strict observational constraints have to be included and explained by theories that model the stellar formation and evolution of stars between 1.5 and $4.5 M_{\odot}$ in the presence of strong magnetic fields.

Acknowledgements. This research was performed within the projects $P 17580$ and $P 17920$ of the Austrian Fonds zur Förderung der wissenschaftlichen Forschung (FwF). Use was made of the SIMBAD database, operated at the CDS, Strasbourg, France and the NASA's Astrophysics Data System.

\section{References}

Abt, H. A., \& Morrell, N. I. 1995, ApJS, 99, 135

Adelman, S. J. 1980, A\&A, 89, 149

Allende Prieto, C., Barklem, P. S., Lambert, D. L., \& Cunha, K. 2004, A\&A, 420, 183

Bagnulo, S., Landstreet, J. D., Lo Curto, G., Szeifert, T., \& Wade, G. A. 2003, A\&A, 403, 645

Bychkov, V. D., Bychkova, L. V., \& Madej, J. 2003, A\&A, 407, 631

Catalano, F. A., \& Leone, F. 1994, A\&AS, 108, 595

Chen, B., Vergely, J. L., Valette, B., \& Carraro, G. 1998, A\&A, 336, 137

Crawford, D. L. 1975, AJ, 80, 955

Crawford, D. L. 1979, AJ, 84, 1858

Dutra, C. M., \& Bica, E. 2000, A\&A, 359, 347

Flower, P. J. 1996, ApJ, 469, 355

Glagolevskij, Yu. V. 1994, Bull. Spec. Astrophys. Obs., 38, 152

Glagolevskij, Yu. V. 2002, Bull. Spec. Astrophys. Obs., 53, 33
Golay, M. 1980, Vistas Astron., 24, 141

Gómez, A. E., Luri, X., Grenier, S., et al. 1998, A\&A, 336, 953

Hauck, B., \& Künzli, M. 1996, Baltic Astron., 5, 303

Hauck, B., \& North, P. 1993, A\&A, 269, 403

Hilditch, R. W., Hill, G., \& Barnes, J. V. 1983, MNRAS, 204, 241

Hubrig, S., North, P., \& Mathys, G. 2000, A\&A, 539, 352

Künzli, M., North, P., Kurucz, R. L., \& Nicolet, B. 1997, A\&AS, 122, 51

Lanz, T. 1984, A\&A, 139, 161

Lutz, Th. E., \& Kelker, D. H. 1973, PASP, 85, 573

Mermilliod, J.-C., Mermilliod, M., \& Hauck, B. 1997, A\&AS, 124, 349

Moss, D. 1989, MNRAS, 236, 629

Napiwotzki, R., Schönberner, D., \& Wenske, V. 1993, A\&A, 268, 653

Nishimura, M., Sadakane, K., Kato, K., Takeda, Y., \& Mathys, G. 2004, A\&A, 420, 673

North, P. 1993, ASPC, 44, 577

North, P. 1998, A\&A, 334, 181

Paunzen, E., Iliev, I. Kh., Kamp, I., \& Barzova, I. S. 2002, MNRAS, 336,1030

Paunzen, E., Stütz, Ch., \& Maitzen, H. M. 2005, A\&A, 441, 631

Pöhnl, H., Maitzen, H. M., \& Paunzen, E. 2003, A\&A, 402, 247

Preston, G. W. 1974, ARA\&A, 12, 257

Rees, D. G. 1987, Foundations of Statistics (London: Chapman \& Hall)

Renson, P. 1991, Catalogue Général des Étoiles Ap et Am, Institut d'Astrophysique, Université Liège

Renson, P., \& Catalano, F. A. 2001, A\&A, 378, 113

Schaerer, D., Meynet, G., Maeder, A., \& Schaller, G. 1993, A\&AS, 98, 523

Schaller, G., Schaerer, G., Meynet, G., \& Maeder, A. 1992, A\&AS, 96, 269

Skiff, A. B. 2003, VizieR On-line Data Catalog: III/233. Originally published in Lowell Observatory (2003)

Smalley, B., \& Kupka, F. 1997, A\&A, 328, 349

Stepien, K. 1994, in Chemically Peculiar and Magnetic Stars, ed. J. Zverko, \& J. Ziznovsky, Slovak Acad. Sci., 8

Wolff, S. C. 1983, The A-type stars: Problems and perspectives, NASA SP-463 